\title{
Biotransformation of Metronidazole by Cunninghamella elegans ATCC 9245
}

\author{
Julia M. Sorrentino*a, Rafaela M. Sponchiado*a, Natália O. dos Santos ${ }^{\mathrm{b}}$, Sendy S. Oliveira ${ }^{\mathrm{b}}$, Karina Galle ${ }^{\mathrm{b}}$, Cássia V. \\ Garcia $^{\mathrm{a}, \mathrm{b}}$, Alexandre M. Fuentefria ${ }^{\mathrm{a}, \mathrm{b}}$ and Martin Steppe ${ }^{\mathrm{a}, \mathrm{b}}$ \\ aPrograma de Pós-graduação em Ciências Farmacêuticas, Universidade Federal do Rio Grande do Sul, Av. Ipiranga \\ 2752, CEP 90610-000, Porto Alegre - RS, Brasil; \\ ${ }^{b}$ Faculdade de Farmácia, Universidade Federal do Rio Grande do Sul, Av. Ipiranga 2752, CEP 90610-000, Porto Alegre \\ - RS, Brasil \\ *Corresponding author: juliamsorrentino@gmail.com
}

\begin{abstract}
Drug biotransformation studies appear as an alternative to pharmacological studies of metabolites, development of new drug candidates with reduced investment as well as the most efficient production of chemical structures involves and drug quality control studies. A wide range of reactions in biotransformations process are catalyzed by microorganisms. Fungi can be considered as a promising source of new biotransformation reactions. The aim of this study was to evaluate the capacity of metronidazole biotransformation through the filamentous fungus Cunninghamella elegans ATCC 9245. The monitoring of metabolite formation was performed by high-performance liquid chromatography (HPLC) coupled to ultraviolet (UV) spectrophotometry. The results of the biotransformation of metronidazole showed drug consumption in culture and formation of four new chromatographic peaks of chemical structures not elucidated. The method showed it became linear over 10-70 $\mu \mathrm{g} / \mathrm{mL}(\mathrm{r}=0.999953)$. Accuracy, precision and stability studies agree with international guidelines. Results are consistent in accordance to the principles of green chemistry as the experimental conditions had a low environmental impact, and little use of environmental harmful solvents.
\end{abstract}

Keywords: biotransformation, HPLC, metabolites, metronidazole

\section{Introduction}

Drug biotransformation is considered as a detoxification process leads to the formation of polar substances or a form easily excreted from the organism. The understanding of drug metabolism plays an important role in the development of new drugs and pharmacological and toxicological studies (1).

Biotransformation is an alternative tool with great potential, especially for the development of sustainable technologies to produce chemicals and drugs, that is, green chemistry. Microbial biotransformations have been extensively employed in the drug discovery and development mainly due to their ability to produce enantioselective products. In addition to that, biotransformation provides an efficient and environmentally friend way to produce large-scale metabolites of drugs and is considered an alternative approach to the asymmetric synthesis and conventional in vivo and in vitro metabolism studies (2-4). This kind of process is attractive for the synthesis of new drug metabolites, which are required for biological and toxicity tests as analytical standards (5-7). Many mammalian phases I metabolic reactions and phase II metabolic reactions, including hydroxylation, oxygen and nitrogen dealkylation, dehydrogenation, and glucuronide and sulfate conjugation, also occur in microbial models.

The filamentous fungi Cunninghamella elegans is useful biocatalysts to modify functional groups, including hydroxylation of aromatic rings, reduction of ketones, oxidations, and demethylations (8-11). Cunninghamella species can metabolize a wide variety of xenobiotics using both phases I and phase II reactions, oftentimes giving rise new compounds $(7,12,13)$.

Several studies have examined biotransformations of drugs by fungi, such as $C$. elegans, demonstrating a broader application to produce drugs metabolites and guiding drug development. Besides that, fungi have not been investigated for possible biotransformation of metronidazole, an antihelminthic drug. Metronidazole (MTZ) is a nitroimidazole antibiotic family and it is widely used in the therapy of several diseases, such as trichomoniasis, amoebiasis and anaerobic infections. The drug is one of the most important agents combined with others antimicrobial therapies used to eradicated Helicobacter pylori, the main cause of gastritis and duodenal ulcers. Studies of metronidazole metabolism in humans have shown the drug is intensively metabolized by the hepatic microsomal cytochrome $\mathrm{P} 450$ with subsequent renal elimination (14). The metabolism of metronidazole is well established in humans (15). However, MTZ-metabolites produced by filamentous fungi are still unknown. The human biotransformation of metronidazole produces three metabolites. The 2-hydroximetronidazole results from the hydroxylation of 2-methyl group, 1-metronidazole acetic acid, from the oxidation of the 1-ethyl group, and glucuronide conjugation on the 1-ethyl group (Figure 1). Both metabolites are found in the urine. Metronidazole and 2hydroximetronidazole had been shown in vitro activity against anaerobic bacteria (16). 


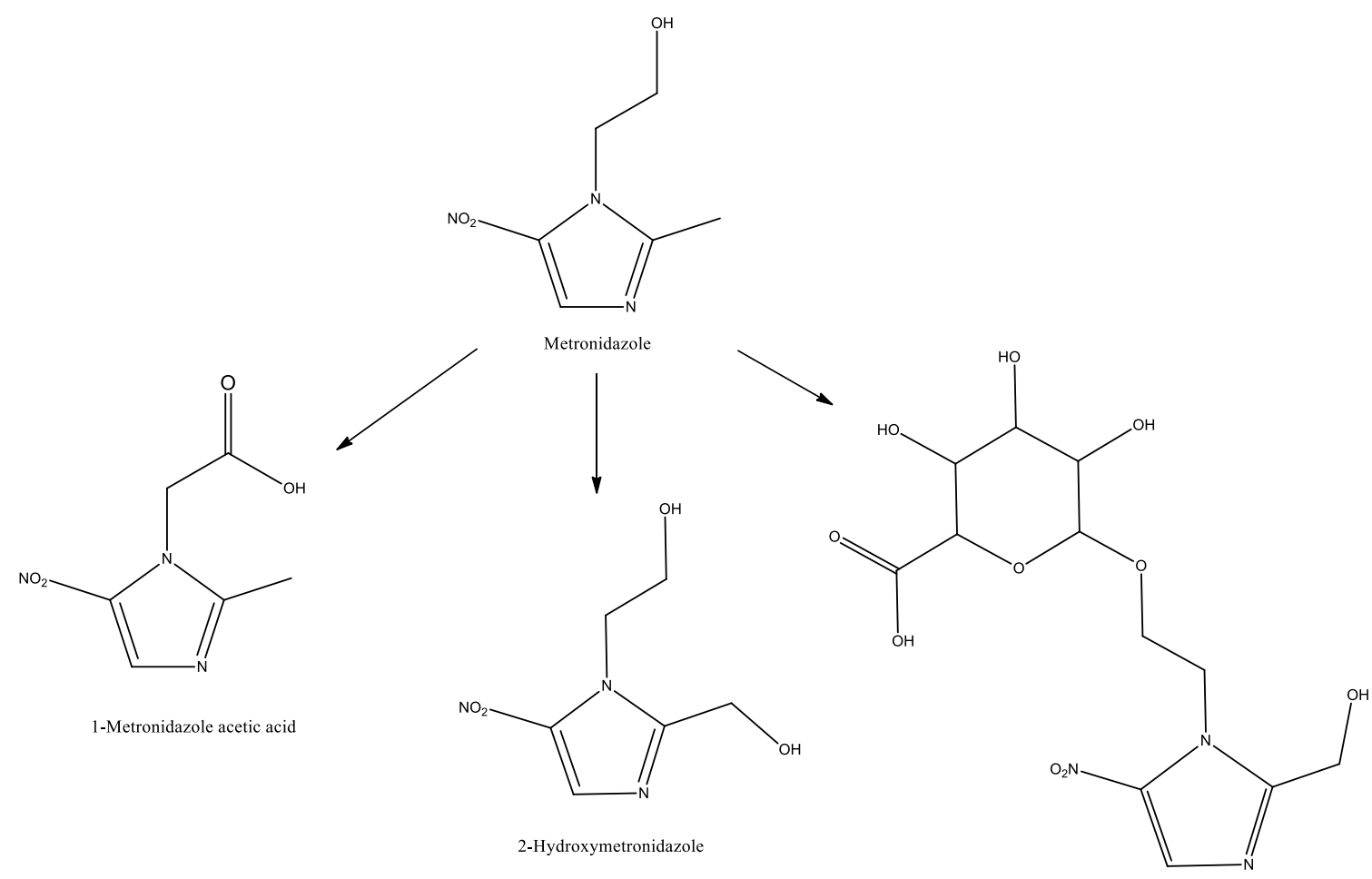

Metronidazole glucuronide

Figure 1 Metronidazole and major metabolites in human urine.

Until now, there have been no reports in the literature of metabolism studies performed with MTZ $(1,12)$. In the light of above considerations, the purpose of this study was first elaborate knowledge of the biotransformation potential of $C$. elegans ATCC 9245 for MTZ and secondly to develop and validate method using HPLC for MTZ analysis in liquid culture medium

\section{Material and methods}

\section{Chemicals}

Methanol and acetonitrile of HPLC grade were purchased from Merck (New Jersey, USA) and Vetec (São Paulo, Brazil), respectively. Czapek broth was obtained from Kasvi (Paraná, Brazil) and Sabouraud dextrose agar (HiMedia, Mumbai, India). Water was purified by a Milli-Q system Millipore (Molsheim, France). Metronidazole was obtained from Farmanguinhos, FIOCRUZ (99.9\%). Dimethylsulfoxide bought from Labsynth (Diadema, São Paulo).

\section{Fungal biotransformation protocol}

C. elegans ATCC 9245 was donated by the Bioconversion Laboratory, Universidade Federal de Goiás (Goiânia, Goiás, Brazil). Stock cultures were cut in discs of $0.5 \mathrm{~cm}$ in diameter of Sabouraud-dextrose agar and maintained on sterile sodium chloride $0.9 \%(\mathrm{w} / \mathrm{v})$ at $5{ }^{\circ} \mathrm{C}$.

\section{Biotransformation}

The biotransformation assay was divided in three stages. The stage I initiated from a standard spore solution of C. elegans. It was prepared by growing the fungi for 3 days at $27^{\circ} \mathrm{C}$ in tubes containing Sabouraud-dextrose agar. A spore suspension was prepared, and $10^{8}$ spores of strain were inoculated in a $250 \mathrm{~mL}$ (17) Erlenmeyer vial containing 150 $\mathrm{mL}$ of a liquid medium with and cultivated for 48 hours at 27 ${ }^{\circ} \mathrm{C}$ in an orbital shaker (New Brunswick ${ }^{\mathrm{TM}}$ Innova ${ }^{\circledR} 2300$ ) at $120 \mathrm{rpm}$.

Stage II was initiated 2 days after inoculation. The substrate MTZ (10.5 mg), which was previously solved in dimethylsulfoxide: water $(1: 1, \mathrm{v} / \mathrm{v})$ was added into each vial containing Czapek broth (drug control, DC) and Czapek broth with spores of $C$. elegans (biotransformation flask, BIO) to give the final MTZ concentration of $70 \mu \mathrm{g} / \mathrm{mL}$. Control samples consisted of sterile culture broth Czapek (blank: B), and culture medium in the presence of fungi (growth control: GC). Both substrate and organism controls were treated under same conditions of incubation.

In stage III, every 24 hours after MTZ addition, $2 \mathrm{~mL}$ aliquot of the liquid culture medium was collected in triplicate (under aseptic conditions). The samples were filtered through nylon membrane $\left(0.45 \mu \mathrm{m}\right.$, Whatman $\left.{ }^{\circledR}\right)$ and stored at low temperature $\left(-20^{\circ} \mathrm{C}\right)$ until the analysis day (8th day).

\section{Chromatographic conditions and apparatus}

Liquid chromatographic analyses of samples were conducted on a Shimadzu LC-10A system equipped with a LC-20AT pump, SPD-20AV UV-VIS variable wavelength detector (313 nm), DGU- 20A5 degasser, CBM-20A controller system, and SIL-20A injection valve with $20 \mu \mathrm{L}$ loop (Kyoto, Japan). The LC Solution v 1.25 software was used for system control.

The chromatographic conditions established were: methanolwater with $0.1 \%$ formic acid $(20: 80, \mathrm{v} / \mathrm{v})$ was chosen as the 
mobile phase, at flow rate $1.0 \mathrm{~mL} / \mathrm{min}$, photodiode array (PDA) detector set at $313 \mathrm{~nm}$. The chromatographic column was a Phenomenex C18 Luna $(250 \times 4.6 \mathrm{~mm}, 5 \mu \mathrm{m})$ and $\mathrm{C} 8$ guard column ( 4 x $3.0 \mathrm{~mm}$; Phenomenex, USA) were kept at $30{ }^{\circ} \mathrm{C}$ and the injection volume was $20 \mu \mathrm{L}$.

\section{Method Validation}

The chromatographic method developed by MTZ in biotransformation assay was validated to certificate the analytical method is singular and trustworthy. The chromatographic method was validated by evaluation of the analytical parameters including specificity, linearity, precision, and accuracy, stability and sensitivity in accordance with FDA - Bioanalytical method validation (18). The recovery, in this study, were not performed.

\section{Specificity}

For the specificity evaluation, the method was performed according to the chromatographic conditions described. The chromatograms of $\mathrm{BIO}, \mathrm{DC}$, and GC were compared to sterile culture (B) and corroborate to the absence of any interference in MTZ peak.

\section{Linearity}

A calibration standard curve was obtained through analysis of solutions obtained by spiking aliquots of Czapek-Dox medium with standard solutions of MTZ concentrations (10 $70 \mu \mathrm{g} / \mathrm{mL}, \mathrm{n}=3$, for each concentration). The calibration curve was evaluated using least-squares linear regression analysis method and ANOVA (analysis of variance) statistical evaluation. The Lower Limit of Quantification (LLOQ) and Limits of Detection (LOD) were estimated according to the standard curve and confirmed through experiments. The reproducibility of the method was analyzed by replicates measurements using the assay.

\section{Precision and Accuracy}

The accuracy and precision of the method were determined by intraday and inter-day ( $\mathrm{n}=3$, for each concentration) assays using Czapek-Dox medium samples spiked with MTZ $(10,40$, and $70 \mu \mathrm{g} / \mathrm{mL})$. Precision was expressed as the relative standard deviation (RSD\%) and the accuracy was expressed as the percent ratio between the experimental concentration and the nominal concentration for each sample.

\section{Stability}

Stability of metronidazole tests was performed in three different ways: (1) freeze and thaw cycle stability and (2) stability at room temperature for $12 \mathrm{~h}$. During freeze and thaw cycle stability, short-term room temperature stability was determined. To perform the freeze and thaw cycle stability, three aliquots of samples, prepared in Czapek-Dox culture medium at the concentration of $70 \mu \mathrm{g} / \mathrm{mL}$ of MTZ were stored at $5{ }^{\circ} \mathrm{C}$ for $24 \mathrm{~h}$ and thawed at room temperature. Then, the samples were refrozen for $12 \mathrm{~h}$ under the same conditions. The freeze and thaw cycle were repeated another two times and samples analyzed after the third cycle. For the determination of the stability at room temperature for $12 \mathrm{~h}$, aliquots of samples prepared in Czapek-Dox culture medium at the concentrations specified previously were kept at $22-$
$25{ }^{\circ} \mathrm{C}$. The concentration obtained from stability tests were compared with recovery concentration determined by freshly prepared samples being considered stable if the deviations (expressed as RSD, \%) observed for the concentrations from the fresh samples were within $<15 \%$. The method was validated according to recommendations of the official guide (18).

\section{Results and discussion}

\section{Fungal biotransformation study}

In general, drug metabolism studies use animal models, such as mice, dogs, and cats $(1,19)$. Those methods suffer from limitations due to the ethical issues, species variation, and cost of experimental animals. Instead of animal models, in vitro and ex vivo studies have been chosen. For this reason, biotransformation using microbial have been explored to the development and drug discovery. The microbial, especially filamentous fungi, in biotransformation studies, represent an alternative to mimic mammalian metabolism $(12,20)$.

The work here presented as a part of our ongoing efforts focused on the development and optimization of biotransformation of drug studies and e validation of the analytical method. In previous studies, several parameters related to the culture medium were modified during the process of biotransformation of drugs, such as the amount of oxygen, $\mathrm{pH}$, temperature, and speed of culture medium shacking $(21,22)$. According to those parameters, modifications were done to optimize de biotransformation process. As results, the better condition was defined, for metronidazole biotransformation, was to maintain the shaken condition at $120 \mathrm{rpm}$, room temperature at $27^{\circ} \mathrm{C}$ and CzapekDox as culture medium.

For monitoring of biotransformation reactions, aliquots were collected every 24 hours, filtered and kept freeze until the analysis by the liquid chromatography method. After 8 days, the HPLC elution profiles of the samples was observed the MTZ biotransformation process showed the production of four products (The most polar substance was eluted in 2.6 minutes, followed by $4.05,4.60$ and 5.20 minutes before then the MTZ, 5.65 minutes (Figure 2). The concentration of MTZ in BIO showed a decrease and, moreover, area peaks of metabolites were increasing during the time assay. Otherwise, the DC concentrations of MTZ had been maintained as the initial time and no different peak chromatographic was observed in the DC. According to this, is possible to infer the metronidazole was metabolized by C. elegans ATCC 9245.

According to the results, the biotransformation reactions of metronidazole by $C$. elegans ATCC 9245 was effective. Those results are consistent with the hypothesis that $C$. elegans can mimic mammalian metabolism of drugs. In fact, this fungus has enzymes that can catalyze this reaction $(12,23)$. Demonstrating the importance and necessity of investment in these studies involving filamentous microorganisms. According to the results of DC, is possible to exclude the drug degradation in Czapek-Dox medium even though it was verified in the bioanalytical validation study Figure 3. 


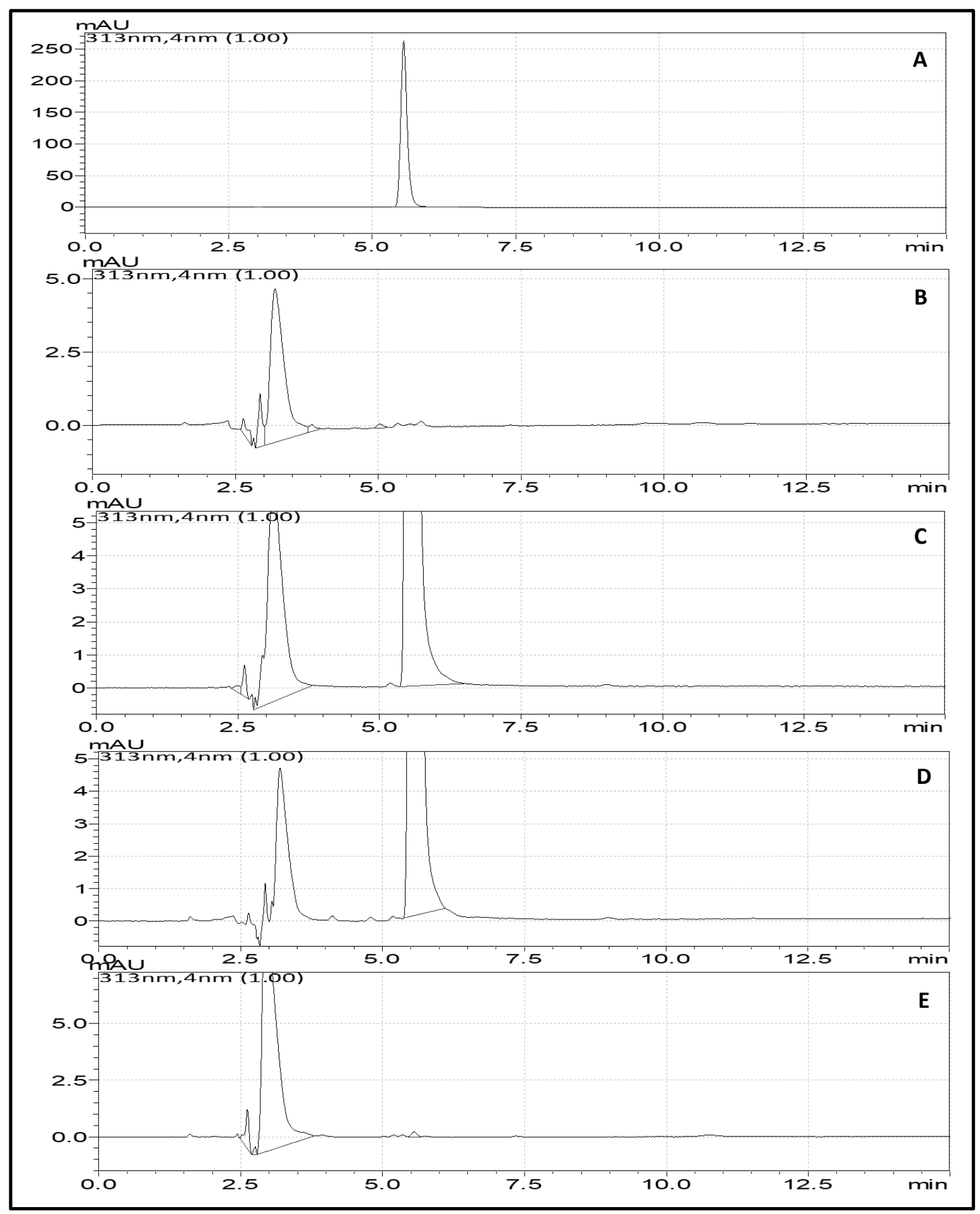

Figure 2 Comparatives chromatograms of metronidazole biotransformation study: A) Standard solution of MTZ (70 $\mu \mathrm{g} / \mathrm{mL})$, B) Growth Control, C) Drug Control, D) Biotransformation and E) Blank. Both samples were incubated for 8 days. 


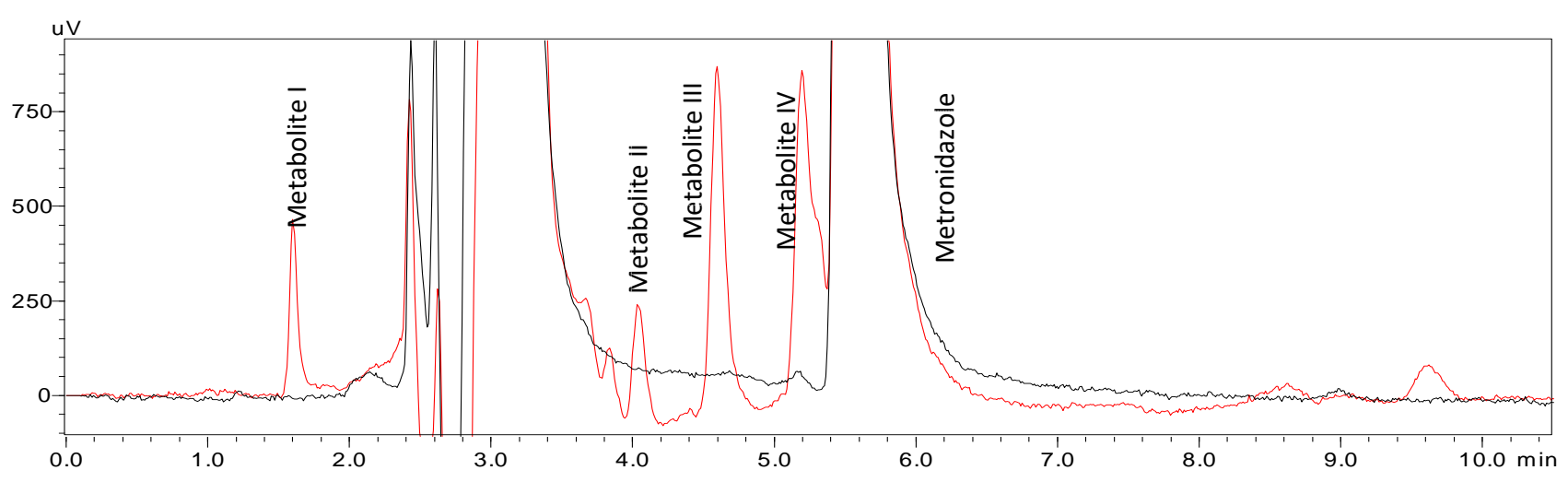

Figure 3 Representative chromatograms of metronidazole after incubation with C. elegans ATCC 9245 for 8 days (red line) and drug control (black line) on enlarged scale.

\section{Method Validation}

Analytical conditions allowed an adequate peak signal of MTZ (6.03 minutes) in the presence of matrix and fungi. Considering the possibility of secondary metabolites and the constituents of the culture medium, a selectivity test was run. However, it was not noted interference peaks into the chromatogram to the analyte and standard.

The linearity of the method was verified by linear regression of the plot of the standard area versus the nominal concentrations of the MTZ. The method was linear over the concentration range of $10-70 \mu \mathrm{g} . / \mathrm{mL}(\mathrm{y}=33129 \mathrm{x}-3597.4, \mathrm{r}$ $=0,999953)$. According to data obtained from ANOVA, it is possible to ensure significant linear regression $\left(\mathrm{F}_{\text {calculated }}=\right.$ $105741.3269>F_{\text {critical }}=6.61$ ) without deviation from linearity $\left(\mathrm{r}^{2}=0.9941\right)$. Limits of detection and lower quantification (LOD and LLOQ) were 0.06 and $0.21 \mu \mathrm{g} / \mathrm{mL}$, respectively. The precision and accuracy of the method were evaluated in terms of MTZ in the culture medium. These results show that the method is precise and accurate within limits appropriate for analytical validation.

Table 1 Calibration curve for the analytical method for metronidazole quantification in Czapek-Dox medium in the range of $10.0 \mu \mathrm{g} / \mathrm{mL}$ to $70 \mu \mathrm{g} / \mathrm{mL}$.

\begin{tabular}{ccccc}
\hline \multirow{2}{*}{$\begin{array}{c}\text { Metronidazole } \\
(\mu \mathrm{g} / \mathrm{mL})\end{array}$} & \multicolumn{2}{c}{ Precision (RSD\%) } & \multicolumn{2}{c}{ Accuracy (\%) } \\
\cline { 2 - 5 } & $\begin{array}{c}\text { Intra- } \\
\text { assay }\end{array}$ & $\begin{array}{l}\text { Inter- } \\
\text { assay }\end{array}$ & $\begin{array}{l}\text { Intra- } \\
\text { assay }\end{array}$ & $\begin{array}{c}\text { Inter- } \\
\text { assay }\end{array}$ \\
\hline 10 & 0.06 & 1.71 & -0.87 & -2.8 \\
40 & 0.13 & 2.99 & -0.54 & -1.5 \\
70 & 0.08 & 0.10 & -0.45 & -0.5 \\
\hline
\end{tabular}

Additionally, the stability at room temperature for $12 \mathrm{~h}$ of MTZ in Czapek-Dox medium showed RSDs lower than 15\%, in this condition MTZ shown to be stable.

\section{Conclusions}

The validation of the bioanalytical method for metronidazole ensured the reliability and credibility of the method developed in this study. The results presented in this study contribute to increasing the knowledge of biotransformation mediated by $C$. elegans ATCC 9245 of drugs in a class little explored. Furthermore, the analytical results of the negative control exclude possible degradation of the drug in the aqueous medium. The validation of the bioanalytical method for metronidazole ensured the reliability and credibility of the bioanalytical method developed in this study, according to normative guides of bioanalytical validation $(18,24)$.

\section{Acknowledgments}

The authors are grateful to Coordenação de Aperfeiçoamento de Pessoal de Nível Superior (CAPES) for financial support and for granting research fellowships.

\section{Conflict of interest}

The authors declare no conflicts of interest.

\section{References}

1. Srisilam K, Veeresham C. Biotransformation of drugs by microbial cultures for predicting mammalian drug metabolism. Biotechnol Adv. 2003;21(1):3-39.

2. Rydevik A. Drug Metabolites Formed by Cunninghamella Fungi. 2014.

3. Pollard DJ, Woodley JM. Biocatalysis for pharmaceutical intermediates: the future is now. Trends Biotechnol. 2007;25(2):66-73.

4. Quinn L, Dempsey R, Casey E, Kane A, Murphy CD. Production of drug metabolites by immobilised Cunninghamella elegans: from screening to scale up. J Ind Microbiol Biotechnol [Internet]. 2015;42(5):799-806.

5. Barth T, Conti R, Pupo MT, Okano LT, Bonato PS. Chiral HPLC analysis of donepezil, 5-O-desmethyl donepezil and 6-O-desmethyl donepezil in culture medium: Application to fungal biotransformation studies. Anal 
Bioanal Chem. 2012;404(1):257-66.

6. de Paula NC, Araujo Cordeiro KCF, de Melo Souza PL, Nogueira DF, da Silva e Sousa DB, Costa MB, et al. Biosynthesis of human diazepam and clonazepam metabolites. Bioorg Med Chem Lett [Internet]. 2015;25(5):1026-9.

7. Baydoun E, Smith C, Wahab A-, Khan MSA, Ahmad MS, Farran D, et al. Microbial transformation of oxandrolone with Macrophomina phaseolina and Cunninghamella blakesleeana. Steroids [Internet]. 2015;102(June):39-45.

8. Williams AJ, Deck J, Freeman JP, Paul Chiarelli M, Adjei MD, Heinze TM, et al. Biotransformation of flumequine by the fungus Cunninghamella elegans. Chemosphere. 2007;67(2):240-3.

9. Keum YS, Lee YH, Jeong-Han K. Metabolism of methoxychlor by cunninghamella elegans ATCC 36112. J Agric Food Chem. 2009;57(17):7931-7.

10. Smith R V., Rosazza JP. Microbial models of mammalian metabolism. Aromatic hydroxylation. Arch Biochem Biophys. 1974;161(2):551-8.

11. Parshikov I a., Sutherland JB. Microbial transformations of antimicrobial quinolones and related drugs. J Ind Microbiol Biotechnol. 2012;39(12):1731-40.

12. Asha S, Vidyathi M. Cunninghamella - A microbial model for drug metabolism studies - A review. Biotechnol Adv [Internet]. 2009 Jan;27(1):16-29.

13. Pearce CM, Lushnikova M V. Microbiological production of omeprazole metabolites by Cunninghamella elegans. J Mol Catal B Enzym. 2006;41(3-4):87-91.

14. Jensen C, Gugler R. Single- and multiple-dose metronidazole. 1983;481-7.

15. Jeelani G, Nozaki T. Entamoeba thiol-based redox metabolism: A potential target for drug development. Mol Biochem Parasitol [Internet]. 2016;206(1-2):39-45.

16. Keefe JPO, Troc KA, Thompson KD. Activity of Metronidazole and its Hydroxy and Acid Metabolites Against Clinical Isolates of Anaerobic Bacteria. 1982;22(3):426-30.

17. Jesus RS. Avaliação da formação de biofilme de fungos emergentes e sua susceptibilidade a antifungicos na forma livre e nanocapsulada. [Porto Alegre]: Universidade Federal do Rio Grande do Sul; 2013.

18. FDA. Guidance for Industry: Bioanalytical Method Validation. U. S. Department of Health and Human Services. Rockville, MD; 2018.

19. Hegazy MEF, Mohamed TA, ElShamy AI, Mohamed AEHH, Mahalel UA, Reda EH, et al. Microbial biotransformation as a tool for drug development based on natural products from mevalonic acid pathway: A review. J Adv Res [Internet]. 2015;6(1):17-33.

20. Murphy CD. Drug metabolism in microorganisms. Biotechnol Lett [Internet]. 2015 Jan;37(1):19-28.

21. Gurram SP, Kollu NR, Sivadevuni G, Solipuram MR. Biotransformation of albendazole by Cunninghamella blakesleeana: Influence of incubation time, media, vitamins and solvents. Iran J Biotechnol. 2009;7(4):20515.

22. Sponchiado R, Sorrentino JM, Olegário N, Oliveira SS, Cordenonsi LM, Silveira GP, et al. Microbial transformation of ambrisentan to its glycosides by Cunninghamella elegans. Biomed Chromatogr. 2019;33(6):1-7.
23. Murphy CD. Drug metabolism in microorganisms. Biotechnol Lett. 2015;37(1):19-28.

24. BRASIL M das S, ANVISA AN de VS. Resolução - RDC $N^{\circ} 27$, de 17 de mao de 2012. Dispõe sobre os requisitos mínimos para validação de métodos bioanalíticos empregados em estudos com fins de registro e pósregistro de medicamentos. [Internet]. Diário Oficial da União. Brasília; 2012. 